\title{
Studies on pre-treatment by compression for wood impregnation I: effects of compression ratio, compression direction, compression speed and compression-unloading place on the liquid impregnation of wood
}

\author{
Youke Zhao ${ }^{1,2}$ (I) Zhihui Wang ${ }^{2} \cdot$ Ikuho lida $^{3} \cdot$ Juan Guo $^{2}$
}

Received: 31 January 2018 / Accepted: 25 April 2018 / Published online: 4 June 2018

(c) The Japan Wood Research Society 2018

\begin{abstract}
To improve the impregnation of wood, the pre-treatment by compression was systematically studied in terms of effects of compression ratio, compression direction, compression speed and compression-unloading place on the liquid impregnation in poplar and Chinese fir. The results showed: the impregnation increased 0.0065 or $0.0074 \mathrm{~g} / \mathrm{cm}^{3}$ for every $1 \%$ increase of compression ratio when the compression ratio was lower or equal to 50 and $40 \%$ for poplar and Chinese fir, respectively; it continued to increase afterwards while the variation was quite big. There existed a significant difference of the impregnation of wood compressed at different directions in Chinese fir, but not in poplar. There existed a significant difference of the impregnation of wood compressed at different speed in both species. The impregnation of wood is likely to be in favor of radial compression in terms of the amount of impregnation. 5 and $10 \mathrm{~mm} / \mathrm{min}$ were recommended as a compromise of impregnation and pre-treatment efficiency. The impregnation of wood that the compression unloaded in water was about 18.2 (poplar) and 9.2\% (Chinese fir) higher in amount and was much quicker in speed than that the compression unloaded in air, and the difference between them was significant, suggesting that compression unloaded in water is significant to improve the impregnation.
\end{abstract}

Keywords Pre-treatment $\cdot$ Compression $\cdot$ Wood impregnation $\cdot$ Penetration

\section{Introduction}

Liquid impregnation is one of the most important operations in wood industry, by which the dimension stability, the strength, the durability, the fire retardant properties, etc. of wood can be improved accordingly with related functionimproved chemicals. However, the improvement is usually

Juan Guo

guojuanchina@126.com

1 Research Institute of Forestry New Technology, Chinese Academy of Forestry, Qing Long Qiao, Dong Xiao Fu No.1, Hai Dian District, Beijing 100091, People's Republic of China

2 Research Institute of Wood Industry, Chinese Academy of Forestry, Qing Long Qiao, Dong Xiao Fu No.1, Hai Dian District, Beijing 100091, People's Republic of China

3 Laboratory of Wood Technology, Kyoto Prefectural University, Shimogamo Nakaragi-cho, Sakyo-ku, Kyoto 606-8522, Japan very limited due to very limited impregnation; therefore, a lot of studies have been done around the improvement of the liquid penetration of wood. Of all the methods studied, the pre-treatment by compression is believed to be one of the most important methods because of high efficiency and easy industrialization compared with other treatments such as microwave treatment, chemical extraction treatment and biological treatment. Pre-treatment by compression is becoming more significant when it comes to a low or no strength loss after the treatment.

Pre-treatment by compression to improve intake of preservatives was as early as around 1970s [1,2] and a rather narrow compression levels at 2.5, 5.0, 7.5, 10.0, 12.5 and $15.0 \%$ were tested in terms of retention, strength and dimension change of spruce wood. More studies around compression treatment for the improvement of liquid penetration have been done in the recent years. Observation by scanning electron microscope (SEM) on the compression wood by Iida [3] found that even at a compression ratio of $68.4 \%$, any 
failure and separation of the cell walls could not be detected, the dimension and cell shapes of the compressed specimens were almost restored to their original state, and the interpretation on the mechanism was given in terms of cellulose, lignin and hemicelluloses. Pit fractures caused by compression explain somehow the mechanism of the improvement of penetration in anatomy aspect [4]. A series studies of liquid penetration of precompressed wood have been done in terms of the effects of sorts of factors on liquid penetration, including the effects of compressive deformation and recovery [5]; thickness and length of specimen [6], moisture content (MC) and temperature while compression [7], cyclic loading, type of impregnated chemicals and annual ring angles [8]; and combined treatment [9] on liquid penetration. All these studies showed the pre-treatment by compression could significantly improve the liquid penetration. In addition to this conclusion, the recovery rate and mechanical properties of pre-compressed wood could be well retained after the treatment [10-12]. The course of liquid uptake of compressed wood by confocal laser scanning microscope [13] showed the uptake of water was detected first between deformed and undeformed regions of compressed wood at all compression ratios tested. For the industrial practice, many researches on roller-pressing method were studied to improve the impregnation of wood [14, 15].

Despite the aforementioned studies, the studies on the improvement of liquid penetration are not enough. Particularly, the effects of compression direction, compression speed and compression-unloading place on the liquid impregnation are not clear. The compression direction determines the way the wood is compressed; the compression speed and the compression ratio directly affect the processing efficiency; the compression unloaded in impregnated solution compared with that in air, as well as the compression in high ratio compared with low ratio, are more difficult in operation, but are assumed more in amount of impregnation; however, it is not clear how big the exact differences are and if the differences are significant. Therefore, the objective of this study is to systematically study the effects of compression ratio, compression direction, compression speed and compression-unloading place on liquid impregnation of wood.

\section{Materials and methods}

15 trees of 25-year-old poplar (Populus tomentosa) plantation with the diameter at the breast height of $25-33 \mathrm{~cm}$ and the air-dried density of $0.43 \mathrm{~g} / \mathrm{cm}^{3}$, and five trees of 25 -yearold Chinese fir (Cunninghamia lanceolata) plantation with the diameter at the breast height of $22-26 \mathrm{~cm}$ and the airdried density of $0.36 \mathrm{~g} / \mathrm{cm}^{3}$ were collected from Guanxian County of Shandong Province and Suichuan County of
Jiangxi Province, respectively. The specimens were prepared with the size of $30 \mathrm{~mm}$ (compression direction) $\times 50 \mathrm{~mm}$ (direction perpendicular to the compression) $\times 100 \mathrm{~mm}$ (longitudinal direction) and tested in radial compression and $45^{\circ}$ compression. Tangential compression was ignored because of obvious shape deformation of wood after the recovery compared with radial and $45^{\circ}$ compression. The radial compression was carried on the flat grain specimens with the compression direction perpendicular to the annual ring; and the $45^{\circ}$ compression was carried on in-between tangential and radial specimens with the compression face having $45^{\circ}$ with the annual ring. The $45^{\circ}$ compression specimens were tested because in the industry practice, most boards are neither vertical nor flat grain, but in-between vertical and flat grain.

To minimize the effects of specimens variation on the test results, all the specimens were oven-dried first so that the growth stress was released in some extent, and then vacuumpressure treated so that all the specimens were fully water saturated having a similar MC, and would be easily compressed. The compression ratio at 10,20, 40, 50 and $60 \%$, and compression speed at $0.5,1,3,5$ and $10 \mathrm{~mm} / \mathrm{min}$ were easily, respectively, controlled by fully computer-controlled Instron 5582 Universal Test Machine, whose compression head was connected with a special adapter. The specimen was put between two stainless plates of the adapter and was compressed by the plate driven by the compression head of the machine. The compression was fixed or released by means of tightening or loosening the nuts on four bolts located at the corner of the adapter. Five repeats (each repeat uses a specimen) for each compression ratio level at given compression speed and direction, as well as for each compression speed level at given compression ratio and direction were tested, respectively.

The impregnation was carried out by free immersion method, which needs the specimens to be totally immersed in water (as the impregnated liquid) during the impregnation. The weight of the wood at different states was measured. The impregnation was finished when the difference of weight in two consecutive measures was less than $0.2 \mathrm{~g}$ in $1 \mathrm{~h}$ and was expressed by the weight gained per unit volume of wet wood.

\section{Results and discussion}

\section{Effects of compression ratio on the impregnation}

To limit the variation, all the specimens for studying the effects of compression ratio on the impregnation were radially compressed at a speed of 3 and $5 \mathrm{~mm} / \mathrm{min}$ for poplar and Chinese fir, respectively. The compression was unloaded in water. 


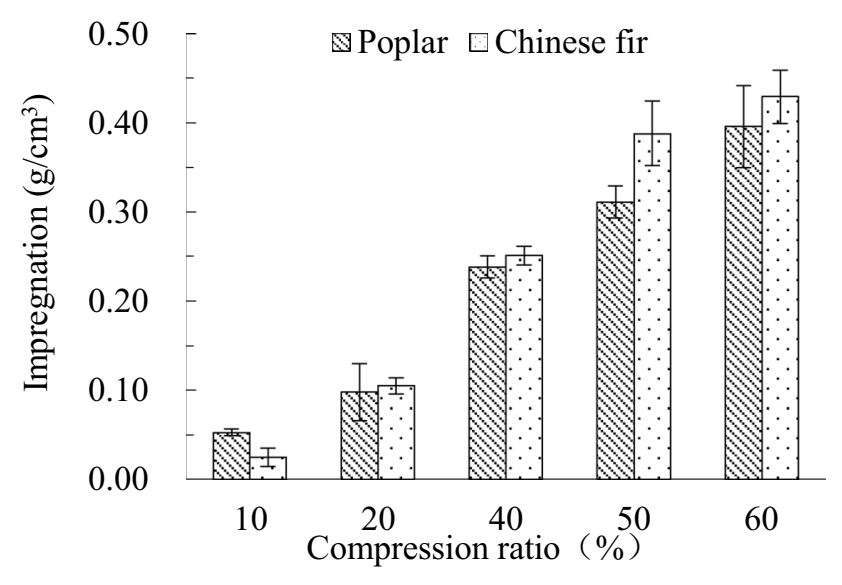

Fig. 1 Impregnation of specimens after the pre-treatment by compression at different compression ratio

Impregnation at different compression ratio (Fig. 1) showed it increased remarkably with the increase of compression ratio. Impregnation was only 0.053 and $0.025 \mathrm{~g} /$ $\mathrm{cm}^{3}$ at $10 \%$ compression ratio, while was 0.400 and $0.429 \mathrm{~g} /$ $\mathrm{cm}^{3}$ at $60 \%$ compression ratio for poplar and Chinese fir, respectively. Compression reduced the wood size along the compression direction, causing the volume shrink during the compression which would resulted in the MC reduction [16] and compression energy was supposed to be stored in wood. With the increase of compression ratio, more space, especially the cavity of vessel in poplar and tracheid lumen in Chinese fir, would be saved for potential impregnation resulted from MC reduction, and more compression energy was supposed to be stored in wood. As soon as the compression was released in water, wood was recovered in a very short time, almost simultaneously by the spring back force of microfibrills, which enlarged the wood cavity volume, and was believed to cause the temporary low pressure within the wood cavity compared with the atmosphere pressure plus the water pressure outside the wood. Under this pressure gradient, the water was absorbed into wood [17]. The bigger the compression ratio was, the more energy was supposed to be stored in wood and more space was saved for the absorption. This explains the results in Fig. 1 why the impregnation increased with the increase of compression ratio.

The absorption of water, especially at low compression ratio, was easily first occurred in vessels rather than fibers in poplar because of the large diameter and the perforate plate in vessels, while that always steadily occurred in lumen in Chinese fir. At high compression ratio, after the release of compression, more water occupied the space in vessels, the absorption in fibers begin to occur, which was harder than that in lumen of tracheid. The impregnation capacity at high compression ratio was determined by the compressible voids since they could be almost totally restored after the release of compression. The voids in Chinese fir was about $10 \%$ more than that in poplar derived from the data of cell wall ratio of both species [18]. That well explained why the impregnations in Chinese fir at all the same compression ratio were higher than those in poplar except that at a low ratio of $10 \%$.

Linear relationship between impregnation and compression ratio (Fig. 2) was found when the compression ratio was lower or equal to 50 and $40 \%$ for poplar and Chinese fir, respectively, suggesting that the impregnation increased 0.0065 or $0.0074 \mathrm{~g} / \mathrm{cm}^{3}$ for every $1 \%$ increase of compression ratio for poplar and Chinese fir, respectively. The impregnation continued to increase afterwards for poplar and Chinese fir, respectively, while the variation was quite big (Fig. 2).

It is worth to notice that, the compression ratio ranges that existed the linear relationship for impregnation for poplar and Chinese fir were exact the same with those existed the linear relationship for recovery rate [11]. Over these ranges, the recovery rate also had a quite big variation. From this, it concludes that the recovery of wood, the intrinsic character of wood, is one of the most important driving forces for the impregnation of wood resulting from extrinsic pre-treatment by compression. The other intrinsic change of wood itself was the pit fractures caused by extrinsic compression [4], resulting in more flow paths for the transportation of water. The more the wood was compressed, the more driving force and flow paths were created.

The above results were based on water saturated condition, the effects of compression on different MC of wood on the impregnation will be report later.

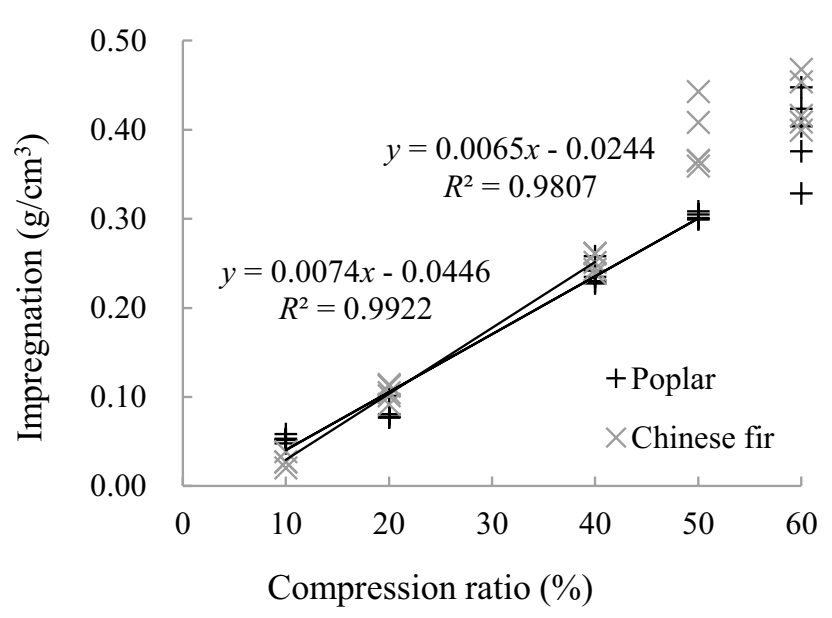

Fig. 2 Linear relations between the impregnation and the compression ratio when the compression ratio $\leq 50 \%$ (poplar) or $\leq 40 \%$ (Chinese fir) 


\section{Effects of compression direction and compression speed on the impregnation}

To limit the variation, all the specimens for studying the effects of compression direction and compression speed on the impregnation were compressed at the compression ratio of 60 and $40 \%$ for poplar and Chinese fir, respectively. The compression was unloaded in water.

Impregnation of wood compressed at different directions and at different speed is shown in Fig. 3. For poplar, the impregnation of wood compressed radially at 0.5 and $1 \mathrm{~mm} /$ min was almost the same or a little bit lower than those compressed $45^{\circ}$, while the impregnation of wood compressed radially at 3,5 and 10 was higher than those compressed $45^{\circ}$. Analysis of variance (ANOVA) (Table 1) showed the difference in impregnation of wood compressed in different directions was not statistically significant in poplar; for Chinese fir, the impregnation of wood compressed radially at all compression speed was higher than that compressed $45^{\circ}$, respectively, and the difference in impregnation of wood compressed in different directions was statistically significant. This can be explained from the early study [11] that, for Chinese fir, the recovery rate of $45^{\circ}$ compression was lower than that of radial compression: the microfibrils were first bent in the corner of near rectangle shaped wood cell when it was compressed $45^{\circ}$; while the microfibrils in radial wall were firstly bent when it was compressed radially. The microfibrils in the corner were more tolerant to this bend than that in radial wall because microfibrils in the corner intrinsically had a sharp bend and therefore were readily used to this sharp bend. Consequently, the wood recovered less, and impregnated less in $45^{\circ}$ compression. Much uneven structure of poplar compared with even structure of Chinese fir may contribute to the impregnation trend of the former more complicated than that of the later. After all, the impregnation was likely to be in favor of radial compression.

Impregnation of wood compressed at different speed did not show a clear pattern although it showed a similar trend
Table 1 ANOVA: Directions versus speed

\begin{tabular}{llrlll}
\hline Source & SS & $d f$ & MS & $F$ & Sig. \\
\hline Poplar & & & & & \\
Directions & 0.0006 & 1 & 0.0006 & 0.7256 & 0.3994 \\
Speed & 0.0245 & 4 & 0.0061 & 8.0559 & $0.0001^{\text {a }}$ \\
Direction $\times$ speed & 0.0010 & 4 & 0.0003 & 0.3400 & 0.8493 \\
Error & 0.0304 & 40 & 0.0008 & & \\
Total & 0.0566 & 49 & & & \\
Chinese fir & & & & & \\
Directions & 0.0207 & 1 & 0.0207 & 28.5267 & $0.0000^{\mathrm{a}}$ \\
Speed & 0.0368 & 4 & 0.0092 & 12.6738 & $0.0000^{\mathrm{a}}$ \\
Direction $\times$ speed & 0.0092 & 4 & 0.0023 & 3.1814 & 0.0232 \\
Error & 0.0291 & 40 & 0.0007 & & \\
Total & 0.0958 & 49 & & & \\
\hline
\end{tabular}

$S S$ sum of square, $d f$ degree of freedom, $M S$ mean square, $F F$ test statistic, Sig. significance

${ }^{a}$ Significant at 0.01 level

between radial compression and $45^{\circ}$ compression. ANOVA (Table 1) showed the difference in impregnation of wood compressed in different speed was statistically significant in both poplar and Chinese fir. As a compromise of impregnation and pre-treatment efficiency, 5 and $10 \mathrm{~mm} / \mathrm{min}$ were recommended for both poplar and Chinese fir.

\section{Effects of the compression-unloading place on the impregnation}

To limit the variation, all the specimens for studying the effects of compression-unloading place on the impregnation were radial compressed at the speed of $5 \mathrm{~mm} / \mathrm{min}$. A compression ratio of 60 and $40 \%$ for poplar and Chinese fir was, respectively, adopted for no or not obvious shape deformation after impregnation.

The impregnation of wood that the compression was unloaded in water was about 18.2 and $9.2 \%$ higher than that
Fig. 3 Impregnation of wood after pre-treatment by compression at different compression directions and different compression speed

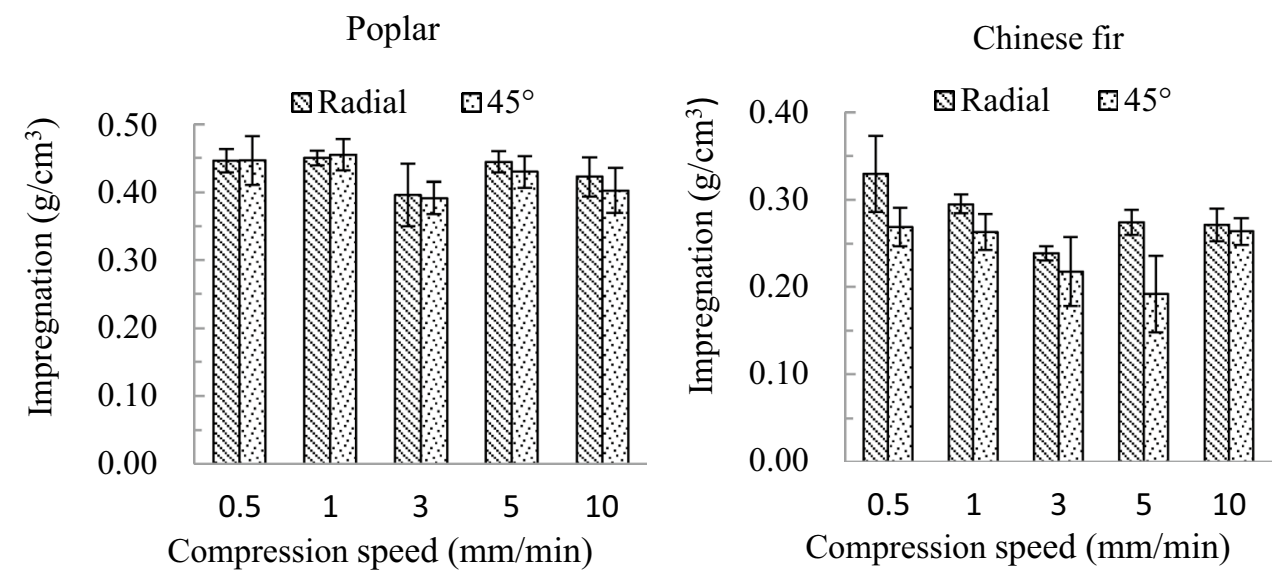




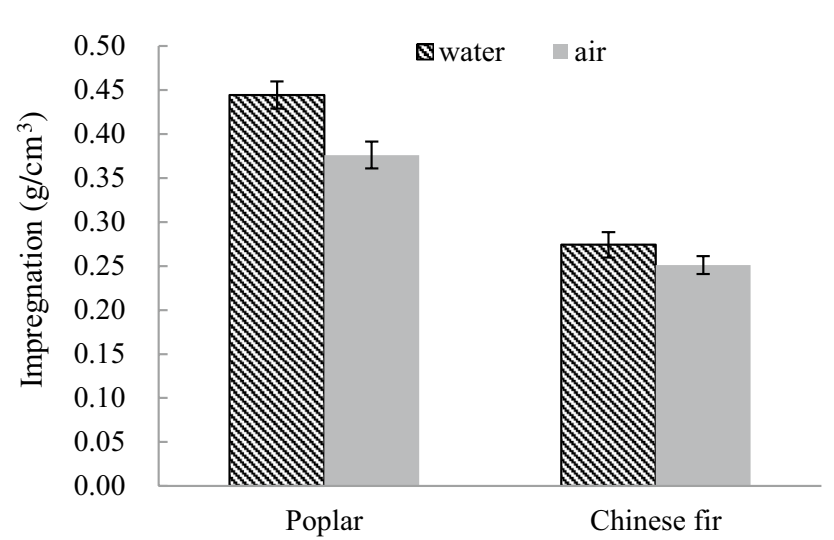

Fig. 4 Impregnation comparison of wood after pre-treatment by compression and unloaded in water and in air

the compression was unloaded in air and then immersed in water for poplar and Chinese fir, respectively (Fig. 4). The former (unloading in water) was 0.44 and $0.27 \mathrm{~g} / \mathrm{cm}^{3}$ and the later (unloading in air) was 0.38 and $0.25 \mathrm{~g} / \mathrm{cm}^{3}$ for poplar and Chinese fir, respectively. Analysis on the variance showed that the difference between them was significant at 0.01 and 0.05 level for poplar and Chinese fir, respectively. It concludes that compression-unloading in water is significant to improve the impregnation.

It was observed that, even the compression was released in a few seconds, the wood was restored to almost the final size in compression direction as soon as the completion of unloading. When it was unloaded in water, quick swollen cell was supposed to cause temporary low pressure within the cell lumen, and therefore would absorb the water into the lumen due to the pressure gradient [17] between pressure outside of wood (atmosphere pressure + water pressure) and inside of wood. It accounted for almost all of the impregnation by the end of the moment of the completion of the unloading in water, since there was almost no more impregnation even after a long time immersing in water. When it was unloaded in air, quick swollen cell was supposed to absorb the air into the lumen in some extent, at least in the cell of outside part of wood. At this condition, the air was believed to deter the impregnation of water, and the impregnation would not become steady until a rather long time period compared with that compression unloaded in water. All these were in favor of the unloading compression in water in terms of the amount of impregnation and the time required for impregnation. Therefore, for the industry, it is recommended to pre-treat the wood by compression and unload the compression directly in the impregnation liquid.

As aforementioned, the voids in Chinese fir were higher than those in poplar. At the same compression ratio, the voids reserved in Chinese fir were supposed to be higher than that in poplar. Higher voids reserved after compression in Chinese fir were considered to have more air stay in wood. When the compression was released, the wood was found to spring back quickly, the water together with the air stayed in wood would redistribute in wood, along with the water impregnating into wood. More air stayed in wood was supposed to have an influence to deter the water absorption even when the compression was unloaded in water. This may explain that the impregnation difference between unloaded in water and in air in Chinese fir was smaller than that in poplar. In this case, lower compression ratio of $40 \%$ for Chinese fir, compared $60 \%$ for poplar, would result in more air to stay in wood, and consequently enlarge the difference of impregnation difference unloaded in water and in air between two species.

\section{Conclusions}

The impregnation increased 0.0065 or $0.0074 \mathrm{~g} / \mathrm{cm}^{3}$ for every $1 \%$ increase of compression ratio when the compression ratio was lower or equal to 50 and $40 \%$ for poplar and Chinese fir, respectively. The impregnation continued to increase afterwards, while the variation was quite big. Impregnations in Chinese fir at all the same compression ratio were higher than those in poplar except that at low ratio of $10 \%$.

There existed a significant difference of impregnation of wood compressed at different directions in Chinese fir, but not in poplar. There existed a significant difference of the impregnation of wood compressed at different compression speed in both poplar and Chinese fir. The impregnation of wood is likely to be in favor of radial compression in terms of the amount of impregnation. 5 and $10 \mathrm{~mm} / \mathrm{min}$ were recommended as a compromise of impregnation and pre-treatment efficiency.

The impregnation of wood that the compression was unloaded in water was about 18.2 (poplar) and 9.2\% (Chinese fir) higher in amount and was much quicker in speed than that the compression was unloaded in air and then impregnated in water, and the difference between them was significant, suggesting that compression unloaded in water is significant to improve the impregnation.

Acknowledgements This work was supported by the Fundamental Research Funds of Research Institute of Forest New Technology, CAF (Grant no. CAFYBB2017SY037).

\section{References}

1. Cech MY, Huffman DR (1970) Dynamic transverse compression treatment of Spruce to improve intake of preservatives. For Prod J 20:47-52 
2. Cech MY (1971) Dynamic transverse compression treatment to improve drying behavior of Yellow Birch. For Prod J 21:41-50

3. Iida I, Norimoto M, Imamura Y (1984) Hygrothermal recovery of compression set (in Japanese). Mokuzai Gakkaishi 30:354-358

4. Watanabe U, Imamura Y, Iida I (1998) Liquid penetration of precompressed wood VI: anatomical characterization of pit fractures. J Wood Sci 44:158-162

5. Iida I, Takayama C, Miyagawa O, Imamura Y (1992) Liquid penetration of precompressed wood I: effects of compressive deformation and recovery upon liquid uptake (in Japanese). Mokuzai Gakkaishi 38:233-240

6. Iida I, Imamura Y, Kashiwa N, Nakamura Y (1992) Liquid penetration of precompressed wood II: effects of thickness and length of specimen on liquid uptake (in Japanese). Mokuzai Hozon (Wood Preserv) 18:31-37

7. Iida I, Ikeuchi A, Imamura Y (1995) Liquid penetration of precompressed woods 3: effects of moisture contents of specimens and ambient temperatures while compression on liquid uptakes of softwoods and hardwoods (in Japanese). Mokuzai Gakkashi 41:811-819

8. Iida I, Mori S, Nakamura Y, Sakai H, Imamura Y (1996) Liquid penetration of precompressed wood V: effects of cyclic loading, type of impregnated chemicals and annual ring angles on the uptake of water or oily solvents (in Japanese). Mokuzai Gakkaishi 42:581-588

9. Iida I, Yusuf S, Watanabe U, Imamura Y (2002) Liquid penetration of precompressed wood VII: the combined treatment of precompression and extraction in hot water on the liquid penetration of wood. J Wood Sci 48:81-85

10. Iida I, Imamura Y (1995) Liquid penetration of precompressed wood 4: mechanical properties of set-fixed wood before and after recovery (in Japanese). Mokuzai Gakkaishi 41:1165-1172
11. Zhao Y, Wang Z, Iida I, Huang R, Lu J, Jiang J (2016) Studies on pre-treatment by compression for wood drying II: studies on pre-treatment by compression for wood drying II: effects of compression ratio, compression direction and compression speed on the recovery rate and mechanical properties of wood. J Wood Sci 62:226-232

12. Zhao Y (2017) Studies on pre-treatment by compression for wood drying III: the reduction of moisture content, the recovery rate, and mechanical properties of wood compressed at different moisture content conditions. J Wood Sci 63:209-215

13. Abe H, Funada R, Kuroda N, Furusawa O, Shibagaki M, Fujii $\mathrm{T}$ (2001) Confocal laser scanning microscopy of water uptake during the recovery of compressed and drying-set wood. Iawa $\mathrm{J}$ 22(1):63-72

14. Adachi K, Inoue M, Kawai S (2005) Liquid impregnation of green wood using a roller-pressing method (in Japanese). Mokuzai Gakkaishi 51:159-165

15. Günzerodt H, Walker JCF, Whybrew K (1988) Compression rolling of sitka spruce and Douglas fir. For Prod J 38:16-18

16. Zhao Y, Wang Z, Iida I, Huang R, Lu J, Jiang J (2015) Studies on pre-treatment by compression for wood drying I: effects of compression ratio, compression direction and compression speed on the reduction of moisture content in wood. J Wood Sci 61:113-119

17. Siau JF (1995) Wood: influence of moisture on physical properties. Department of Wood Science and Forest Products, Virginia Polytechnic Institute and State University, Blacksburg

18. Jiang X, Yin Y (2008) Quantative anatomy of Chinese fir and poplar. In: Jiang Z, Jiang X (eds) Wood structures and their relations with the wood properties (in Chinese). Science Press, Beijing, pp 81-82 